\title{
RELATIVE PROFILES AND EXTENDED WEIGHT POLYNOMIALS OF ALMOST AFFINE CODES
}

\author{
TRYGVE JOHNSEN AND HUGUES VERDURE
}

\begin{abstract}
In this paper we study various aspects concerning almost affine codes, a class including, and strictly larger than, that of linear codes. We use the combinatorial tool demi-matroids to show how one can define relative length/dimension and dimension/length profiles of flags(chains) of almost affine codes. In addition we show two specific results. One such result is how one can express the relative length/dimension profiles (also called relative generalized Hamming weights) of a pair of codes in terms of intersection properties between the smallest of these codes and subcodes of the largest code. The other result tells how one can find the extended weight polynomials, expressing the number of codewords of each possible weight, for each code in an infinite hierarchy of extensions of a code over a given alphabet.
\end{abstract}

Key words: Relative profiles, Almost affine codes, Extended weight polynomials

\section{INTRODUCTION}

In this article we will study various properties of almost affine codes. Such codes were defined in [16]. It is well known ([16]) that $C$ defines a matroid $M_{C}$ through the rank function

$$
\rho(X)=\log _{|A|}\left|C_{X}\right| \text {. }
$$

We will perform a study and concretization of relative profiles of pairs of almost affine codes, as done for example in [14] for pairs of linear codes, as a generalization of profiles for single linear codes in [3]. Since our methods work just as well for flags $F=\left(C_{1}, \cdots, C_{m}\right)$, for any natural number $m$, we will also study relative profiles of such flags for general $m$. Our main tools for doing this will be the theory of demi-matroids as described in [1], and in particular in [2], where profiles of demi-matroids were introduced, taylor-made for studying relative profiles of flags of linear codes. We here extend this study to flags of almost affine codes.

After recalling some basic properties of matroids, demi-matroids and almost affine codes in Section 2, and linking the two topics through Theorem 2.11, taken from [11], we will give the main results of the paper in sections 3 and 4 . First we give a detailed description of relative profiles (including RLDP, RDLP, IRLDP) for flags of almost affine codes, and give a result (Proposition 3.10) about their properties. A main point is to show how much of the material from [3] (linear codes), [14](pairs of linear codes), [2] (flags of linear codes of arbitrary length) that can be generalized to flags of almost affine codes of arbitrary length $m$. Such codes do not even have natural dual codes in general, in contrast to linear codes, and therefore it is a challenge to handle them, but the usage of demi-matroids, that do indeed have dual objects (in two ways) makes it relatively effortless to perform the described generalization, and through Subsection 3.2 we demonstrate this. Furthermore we perform a special study of relative length/dimension profiles for the case $m=2$, that is of pairs of

Date: March 26, 2020.

1991 Mathematics Subject Classification. 94B05. 
JOHNSEN AND VERDURE

almost affine codes, $C_{2} \subset C_{1}$. This is done in Section 4 . We investigate to which extent it is possible to generalize the results in [13] and [18]. There one expresses these relative length/dimension profiles as the minimum weights of subcodes of $C_{1}$ of various dimensions, intersecting $C_{2}$ only in the zero element. In one of our two main results, Theorem 4.1, we show an analogue of this result for almost affine codes. In Remark 4.2 we show, however, that the situation is not completely like in the case of linear codes.

In the last part, Section 5 we study another aspect of the relationship between almost affine codes and matroids: In [7], and [6, p. 323], one points out that for linear block codes of length $n$ over a finite field $\mathbb{F}_{q}$, one can produce an infinite series of codes by extending the alphabet to $\mathbb{F}_{q^{s}}$, for $s=1,2, \cdots$, and nevertheless find polynomials $A_{0}, \cdots, A_{n}$, such that $A_{j}\left(q^{s}\right)$ computes the number of codewords of weight $j$, for all $s$ simultaneously, for each of $j=0, \cdots, n$. We will show that a corresponding result holds for almost affine codes. A main point is that the polynomials $A_{j}$ are only dependent on the assosiated matroid of $C$ in the linear case, and that we have matroids that are equally simple to handle in the general case of almost affine codes. We give an example where we calculate the $A_{j}$ for a non-vectorial almost affine code whose associated matroid is the non-Pappus matroid on 9 elements.

Remark 1.1. This work is an extension of material presented at the "5th International Castle Meeting on Coding Theory and Applications", and published in the proceedings of that conference, [12], and therefore partly overlaps with that paper.

\section{Preliminaries}

2.1. Matroids and demi-matroids. A matroid is a combinatorial structure that extends the notion of linear (in)dependency. There are many equivalent definitions, see [15], but we will give just one here.

Definition 2.1. A matroid is a pair $M=(E, \rho)$ where $E$ is a finite set, and $\rho$ an integer function $\rho: 2^{E} \rightarrow \mathcal{N}$ satisfying the following axioms:

(R1): $\rho(\emptyset)=0$,

(R2): for every subset $X \subset E$ and $x \in E, \rho(X) \leqslant \rho(X \cup\{x\}) \leqslant \rho(X)+1$,

(R3): for every $X \subset E$ and $x, y \in E$, if $\rho(X)=\rho(X \cup\{x\})=\rho(X \cup\{y\})$, then $\rho(X \cup\{x, y\})=\rho(X)$.

Any $M=(E, \rho)$ satisfying (R1) and (R2) is called a demi-matroid. See [1] and [4, Theorem 5.5]. The set $E$ is called the ground set, and the function $\rho$ the rank function.

Definition 2.2. The dual (demi-) matroid is $M^{*}=\left(E, \rho^{*}\right)$, where for any $X \subset E$.

$$
\rho^{*}(X)=|X|+\rho(E-X)-\rho(E) .
$$

In [1] one describes the supplement (or second) dual $(E, \bar{\rho})$ of demi-matroids:

Definition 2.3. Let $s: 2^{E} \rightarrow \mathcal{N}$ be an integer function. For $X \subset E$, define

$$
\bar{\rho}(X):=\rho(E)-\rho(E-X) .
$$

In [1] one also shows that $(E, \bar{\rho})$ is a demi-matroid if $(E, \rho)$ is so.

2.2. Almost affine codes. Almost affine codes were first introduced in [16].

Definition 2.4. An almost affine code on a finite alphabet $A$, of length $n$ and dimension $k$ is a subset $C \subset A^{n}$ such that $|C|=|A|^{k}$ and such that for every subset $X \subset E=\{1, \cdots, n\}$,

$$
\log _{|A|}\left|C_{X}\right| \in \mathcal{N}
$$


where $C_{X}$ is the puncturing of $C$ with respect to $E X$.

An almost affine subcode of $C$ is a subset $D \subset C$ which is itself an almost affine code on the same alphabet.

Remark 2.5. Any linear or affine code is obviously an almost affine code.

To any almost affine code $C$ of length $n$ and dimension $k$ on the alphabet $A$, we can associate a matroid $M_{C}=(E, \rho)$ where for any $X \subset E$

$$
\rho(X)=\log _{|A|}\left|C_{X}\right| \text {. }
$$

Definition 2.6. Let $C$ be an almost affine code of length $n$, and let $c \in C$ be fixed. Let

$$
C(X, \boldsymbol{c})=\left\{\boldsymbol{w} \in C, \boldsymbol{w}_{X}=\boldsymbol{c}_{X}\right\},
$$

where $\boldsymbol{w}_{X}$ is the projection of $\boldsymbol{w}$ to $X$. A subcode of the form $C(X, \boldsymbol{c})$ is called a standard subcode.

By ([16, Corollary 1]) we have:

Proposition 2.7. $C(X, c)$ is an almost affine subcode of $C$ of dimension $\operatorname{dim} C-r(X)$.

Remark 2.8. In other terms $C(X, c)$ is a shortening of $C$ in the coordinates corresponding to $X$. By choosing $X=E_{j}=\{1, \cdots, j\}$, for all possible $j$, one sees that an almost affine code $C$ of dimension $k$ has almost affine subcodes of dimension $0 \leqslant i \leqslant k$. In general, however, standard subcodes are not the only subcodes of almost affine codes.

The following concept will be useful for almost affine codes, which in contrast to linear codes, have no "canonical" reference word $\mathbf{0}$.

Definition 2.9. Let $C$ be a block code of length $n$, and let $\boldsymbol{c} \in C$ be fixed. The c-support of any codeword $\boldsymbol{w}$ is

$$
\operatorname{Supp}(\boldsymbol{w}, \boldsymbol{c})=\left\{i, \boldsymbol{c}_{i} \neq \boldsymbol{w}_{i}\right\}
$$

The c-support of $C$ is

$$
\operatorname{Supp}(C, \boldsymbol{c})=\bigcup_{\boldsymbol{w} \in C} \operatorname{Supp}(\boldsymbol{w}, \boldsymbol{c}) .
$$

Note that the $\boldsymbol{c}$-support of the code is independent of the choice of $\boldsymbol{c} \in C$ (see [9, Lemma $1]$ ), and it will therefore be denoted by $\operatorname{Supp}(C)$ without reference to any codeword. The cardinality of $\operatorname{Supp}(C)$ will be denoted by $w(C)$.

Definition 2.10. A flag $F=\left(C_{1}, \cdots, C_{m}\right)$ of almost affine codes is a finite set of almost affine codes on the same alphabet and of the same length, with the property that for $1 \leqslant j \leqslant$ $m-1, C_{j+1}$ is an almost affine subcode of $C_{j}$. A pair of almost affine codes is a flag with two codes.

From [11] we have:

Theorem 2.11. Let $F=\left(C_{1}, \cdots, C_{m}\right)$ be a flag of $F$ of almost affine codes. Then for

$$
\rho_{F}(X)=\sum_{i=1}^{m}(-1)^{i+1} \rho_{i}(X)
$$

the pair $D=\left(E, \rho_{F}\right)$ is a demi-matroid.

Let $\operatorname{dim} C_{i}=r_{i}$, for $i=1, \cdots, m$. Denote the rank $\sum_{i=1}^{m}(-1)^{i+1} r_{i}$ of the demi-matroid by $R_{F}$. 


\section{Profiles of almost AfFine Codes And Flags}

3.1. Definition via the associated demi-matroid or matroid. Let $D=(E, \rho)$ be a demi matroid. In [2, Definition 13] one defined demi-matroid profiles inspired by profiles from codes:

Definition 3.1. For all $0 \leqslant i \leqslant n, 0 \leqslant j \leqslant k$ and $0 \leqslant l \leqslant n-k$,

$$
\begin{gathered}
k_{i}:=\max \{\bar{\rho}(X):|X| \leqslant i\} \\
\tilde{k}_{i}:=\min \{\rho(X):|X| \geqslant i\} \\
l_{i}:=\max \left\{\overline{\rho^{*}}(X):|X| \leqslant i\right\} \\
\tilde{l}_{i}:=\min \left\{\rho^{*}(X):|X| \geqslant i\right\} \\
m_{j}:=\min \left\{|X|: X \subset E, \bar{\rho}(X)=|X|-\rho^{*}(X) \geqslant j,\right\} \\
n_{l}:=\min \left\{|X|: X \subset E, \overline{\rho^{*}}(X)=|X|-\rho(X) \geqslant j\right\}, .
\end{gathered}
$$

Before giving concretizations of these profiles in the next subsection, for flags of almost affine codes. we first give the following:

Definition 3.2. For $C$ a block code, and $S \subset C$, we set $w t(S)=|\operatorname{Supp}(S)|$. Moreover, for $C$ be a linear code $[n, k]$-code, the generalized Hamming weights are

$$
d_{i}(C)=\min \{w t(D): D \subset C \text { is a subcode of dimension } i,\}
$$

for $i=1, \cdots, k$.

In [9, Definition 1], one defined the generalized Hamming weights of a matroid by

Definition 3.3. Let $M$ be a matroid. For $1 \leqslant i \leqslant n-k$, the $i$-th generalized Hamming weight of $M$ is

$$
d_{i}(M)=\min \{X: X \subset E,|X|-\rho(X)=i\}
$$

As shown in [9], it is a proper generalization of the generalized Hamming weights for linear codes, since we have

$$
d_{i}(C)=d_{i}\left(M_{C^{*}}\right)
$$

for the matroid associated with (the rank function of) the dual code $C^{*}$. In [10], we show that we can define generalized Hamming weights for almost affine codes via its associated matroid, namely

Definition 3.4. Let $C$ be an almost affine code. For $1 \leqslant i \leqslant k$, the $i$-th generalized Hamming weight of $C$ is

$$
d_{i}(C)=d_{i}\left(M_{C^{*}}\right)=\min \left\{|X| ;|X|-\rho^{*}(X)=\bar{\rho}(X)=i\right\} .
$$

In [10, Proposition 4] one stated and proved the following result:

Proposition 3.5. Let $C$ be an almost affine code. Let $\boldsymbol{c} \in C$ be any codeword. Then for every $1 \leqslant i \leqslant k$,

$$
\begin{aligned}
d_{i}(C) & =\min \{|X| ; \rho(E X)=k-i\} \\
& =n-\max \{|X| ; \rho(X)=k-i\} \\
& =n-\max \left\{|X| ;|C(X, \boldsymbol{c})|=|A|^{i}\right\} .
\end{aligned}
$$

The third equality is independent of the choice of $\boldsymbol{c}$. 
We will use Proposition 3.5 as a point of departure for studying relative length/dimension profiles of flags of almost affine codes in Subsection 3.2. The following result was also proved in [10], linking the definition of generalized Hamming weights for almost affine codes to an analogue of the "classical" definition of generalized Hamming weights for linear codes.

Theorem 3.6. Let $C$ be an almost affine code. Then the generalized Hamming weights for $C$ are

$$
\begin{aligned}
d_{i}(C) & =\min \{|\operatorname{Supp}(D)| ; D \text { is an almost affine subcode of dim. } i \text { of } C\} \\
& =\min \{|\operatorname{Supp}(D)| ; D \text { is a standard subcode of dim. } i \text { of } C\} \\
& =n-\max \left\{|X| ;|C(X, \boldsymbol{c})|=q^{i}\right\},
\end{aligned}
$$

for $1 \leqslant i \leqslant k$.

3.2. Profiles in practice - flags of codes. In this subsection we will study the demimatroid $D_{F}$, where

$$
\rho_{F}(X)=\sum_{i=1}^{m}(-1)^{i+1} \rho_{i}(X),
$$

for a flag $F=\left(C_{1}, \cdots, C_{m}\right)$ of almost affine codes. We will now interpret the demi-matroid profiles described above in the case of flags of almost affine codes:

Proposition 3.7. Let $F=\left(C_{1}, \cdots, C_{m}\right)$ be a flag of almost affine codes. Let $\boldsymbol{c} \in C_{m}$. For the demi-matroid $D_{F}$, we have:

$$
\begin{gathered}
\rho_{F}(X)=\sum_{i=1}^{m}(-1)^{i} \operatorname{dim}\left(C_{i}\right)_{X} . \\
\rho_{F}^{*}(X)=|X|+\sum_{i=1}^{m}(-1)^{i}\left(r_{i}-\rho_{i}(E X)\right), \text { and } \\
m_{j}=\min \left\{|X| ; \sum_{i=1}^{m}(-1)^{i+1} \operatorname{dim} C_{i}(E X, \boldsymbol{c}) \geqslant j\right\}, \\
n_{j}=\min \left\{|X| ;|X|+\sum_{i=1}^{m}(-1)^{i} \operatorname{dim}\left(C_{i}\right)_{X} \geqslant j\right\},
\end{gathered}
$$

Moreover:

$$
\begin{gathered}
k_{j}=\max \left\{\sum_{i=1}^{m}(-1)^{i+1} \operatorname{dim} C_{i}(E X, \boldsymbol{c}) ;|X| \leqslant j\right\}, \\
\tilde{k}_{j}=\min \left\{\sum_{i=1}^{m}(-1)^{i+1} \operatorname{dim}\left(C_{i}\right)_{X} ;|X| \geqslant j\right\}, \\
l_{j}=\max \left\{|X|+\sum_{i=1}^{m}(-1)^{i} \operatorname{dim}\left(C_{i}\right)_{X} ;|X| \leqslant j\right\}, \\
\tilde{l}_{j}=\min \left\{|X|+\sum_{i=1}^{m}(-1)^{i} \operatorname{dim} C_{i}(E X, \boldsymbol{c}) ;|X| \geqslant j\right\} .
\end{gathered}
$$

All inequalities above can be replaced by equalities.

Proof. A straightforward insertion of the expression for $\rho(X)$, combined with repeated usage of Proposition 2.7. Changing inequalities into equalities is a standard procedure in (demi)matroid theory since all the functions in question have unique rank increase. 
In [2, Definition 3] and [2, Definition 6] one also defined demi-matroid profiles, which in the notation there were called: $\left\{s_{i}\right\},\left\{t_{i}\right\},\left\{\bar{\sigma}_{i}\right\},\left\{\bar{\tau}_{i}\right\}$ and $\left\{s_{i}\right\},\left\{t_{i}\right\},\left\{\bar{\sigma}_{i}\right\},\left\{\bar{\tau}_{i}\right\}$, One could give corresponding concretizations of those profiles.

We will now study the invariant $m_{j}$ for flags of almost affine codes. A change of variables in the expression of $m_{j}$ in Proposition 3.7 gives:

$$
m_{j}=n-\max \left\{|X| ; \sum_{i=1}^{m}(-1)^{i+1} \operatorname{dim}\left(C_{i}(X, \boldsymbol{c}) \geqslant j\right\} .\right.
$$

We observe that in the case $m=1$ this is the same expression as the last one in Proposition 3.5 for the generalized Hamming weight $d_{j}$ of a single almost affine code $C_{1}$. Moreover, for linear codes, the $d_{j}\left(C^{*}\right)$ for the orthogonal complement $C^{*}$ coincide with the $n_{j}$, correspondingly. The set of generalized Hamming weights for linear codes are frequently called the length/dimension profile (LDP) of the code (See e.g. [3]), and in case of pairs of linear codes (see [3]) it is called the relative length/dimension profile (RLDP).

We thus define:

Definition 3.8. The relative length/dimension profile of a flag $F=\left(C_{1}, \cdots, C_{m}\right)$ of almost affine codes is given by the numbers $m_{1}(F), \cdots, m_{R_{F}}(F)$ :

$$
m_{j}(F)=n-\max \left\{|X| ; \sum_{i=1}^{m}(-1)^{i+1} \operatorname{dim}\left(C_{i}(X, \boldsymbol{c})\right) \geqslant j\right\} .
$$

We observe that in the case $m=1$ this is the same expression as the last one in Proposition 3.5 for the generalized Hamming weight $d_{j}$ of a single almost affine code $C_{1}$. Moreover, for linear codes, the $d_{j}\left(C^{*}\right)$ for the orthogonal complement $C^{*}$ coincide with the $n_{j}$, correspondingly. The set of generalized Hamming weights for linear codes are frequently called the length/dimension profile (LDP) of the code (See e.g. [3]), and in case of pairs of linear codes (see [3]) it is called the relative length/dimension profile (RLDP). In Section 4 we will give a reinterpretation of the $m_{i}(F)$ for the case $m=2$, that is pairs $\left(C_{1}, C_{2}\right)$ of almost affine codes.

Furthermore we will focus on the invariant $k_{j}$ and $\tilde{k}_{j}$ for flags $F=\left(C_{1}, \cdots, C_{m}\right)$ of almost affine codes. The formula

$$
k_{j}=\max \left\{\sum_{i=1}^{m}(-1)^{i+1} \operatorname{dim} C(E X, \boldsymbol{c}) ;|X| \leqslant j\right\},
$$

reduces to:

$$
k_{j}=\max \{\operatorname{dim} C(E X, \boldsymbol{c}) ;|X| \leqslant j\},
$$

for a single code, and the set of these invariants are frequently called the dimension/length profile (DLP) of the code (see e.g. [3] again), and in case of pairs of linear codes it is called (see [3] again) the relative dimension/length profile (RDLP). Likewise the formula:

$$
\tilde{k}_{j}=\min \left\{\sum_{i=1}^{m}(-1)^{i+1} \operatorname{dim}\left(C_{i}\right)_{X} ;|X| \geqslant j\right\}
$$

reduces to:

$$
\tilde{k}_{j}=\min \left\{\operatorname{dim} C_{X} ;|X| \geqslant j\right\},
$$

which is frequently called the inverse distance/length profile (IDLP).

We thus define: 
Definition 3.9. The relative dimension/length profile (RDLP) and the inverse relative length/dimension profiles (IRDLP) of a flag $F=\left(C_{1}, \cdots, C_{m}\right)$ of almost affine codes are the numbers $k_{0}(F), \cdots, k_{n}(F)$, and $\tilde{k}_{0}(F), \cdots, \tilde{k}_{n}(F)$, respectively, and the $k_{j}(F)$ and the $\tilde{k}_{j}(F)$ are given by the formulas

$$
\begin{gathered}
k_{j}(F)=\max \left\{\sum_{i=1}^{m}(-1)^{i+1} \operatorname{dim} C_{i}(E-X, \boldsymbol{c}) ;|X| \leqslant j\right\}, \\
\tilde{k}_{j}(F)=\min \left\{\sum_{i=1}^{m}(-1)^{i+1} \operatorname{dim}\left(C_{i}\right)_{X} ;|X| \geqslant j\right\} .
\end{gathered}
$$

The general results about demi-matroids in [2, Proposition 15] and [2, Proposition 16] now immediately give some results relating these profiles of flags of almost affine codes:

Proposition 3.10. For a flag $F=\left(C_{1}, \cdots, C_{m}\right)$ of almost affine codes we have:

- $k_{i}(F)=\max \left\{j ; m_{j}(F) \leqslant i\right\}$, for $0 \leqslant i \leqslant n$.

- $m_{j}(F)=\min \left\{i ; k_{i}(F) \geqslant j\right\}$, for $1 \leqslant j \leqslant R_{F}$.

- $k_{i}(F)+\tilde{k}_{n-i}(F)=R_{F}$, for $0 \leqslant i \leqslant n$.

- $0 \leqslant k_{i+1}(F)-k_{i}(F) \leqslant 1$, for $0 \leqslant i \leqslant n-1$.

- $0 \leqslant \tilde{k}_{i+1}(F)-\tilde{k}_{i}(F) \leqslant 1$, for $0 \leqslant i \leqslant n-1$.

- $k_{0}(F)=\tilde{k}_{0}(F)=0$, and $k_{n}(F)=\tilde{k}_{n}(F)=R_{F}$

- $m_{j+1}(F) \geqslant m_{j}(F)+1$, for $1 \leqslant j \leqslant R_{F}-1$.

\section{Equivalent formulations of PROFILES For Pairs of CODES}

Let $F=\left(C_{1}, C_{2}\right)$ be a pair of linear codes, and focus on the demi-matroid $D_{F}$. We will give a new interpretation of $m_{i}(F)$ given in Definition 3.8, for $1 \leqslant i \leqslant R_{F}$, which says:

$$
m_{i}(F)=\min \left\{|X| ; \overline{\rho_{F}}(X)=\sum_{j=1}^{m}(-1)^{j+1} \operatorname{dim} C_{j}(E X, \boldsymbol{c}) \geqslant i\right\}
$$

(where $\boldsymbol{c} \in C_{2}$, and where we recall that the $m_{i}(F)$ are equal to the Hamming weights $d_{1}, \cdots, d_{r_{1}}$, of $C_{1}$ in case $C_{2}=0$.) In [18, Propositions 2 and 4], two equivalent ways of expressing the $m_{i}(F)$ are presented. We now will discuss the possibility of expressing the $m_{i}(F)$ in analogous ways, not only for linear codes, but for almost affine codes in general. Our result is given in the theorem below.

Theorem 4.1. Let $F=\left(C_{1}, C_{2}\right)$ be a pair of almost affine codes with associated demimatroid $D_{F}$. Then for $0 \leqslant i \leqslant R_{F}$,

$m_{i}=\min \left\{w(D) ;\left|D \cap C_{2}\right|=1, D \subset C_{1}\right.$ is a standard subcode with $\left.\operatorname{dim} D=i\right\}$.

Proof. Let $b_{i}$ be the right hand side of the above equality. We fix $\boldsymbol{v} \in C_{2}$.

Let $X \subset E$ be such that $|X|=m_{i}$ and $\bar{s}(X)=i$, that is

$$
r_{1}-\rho_{1}(E X)-r_{2}+\rho_{2}(E X)=i
$$

or equivalently

$$
\operatorname{dim} C_{1}(E X, \boldsymbol{v})-\operatorname{dim} C_{2}(E X, \boldsymbol{v})=i .
$$

We have the obvious inclusions

$$
\operatorname{Supp}\left(C_{2}(E X, \boldsymbol{v})\right) \subset \operatorname{Supp}\left(C_{1}(E X, \boldsymbol{v})\right) \subset X .
$$


We claim that the second inclusion is actually an equality. Indeed, if not, let $y \in X$ $\operatorname{Supp}\left(C_{1}(E X, \boldsymbol{v})\right)$ and consider $Y=X\{y\}$. Then, for $j=1,2$, if $\boldsymbol{w} \in C_{j}(E X, \boldsymbol{v}), \boldsymbol{w}_{y}=\boldsymbol{v}_{y}$ since $y$ is not in the support, and in turn, the natural inclusions

$$
C_{j}(E Y, \boldsymbol{v}) \subset C_{j}(E X, \boldsymbol{v})
$$

are equalities. This contradicts the minimality of $X$ since $Y$ also satisfies $\overline{\rho_{F}}(Y)=\overline{\rho_{F}}(X)=$ $i$.

Let $Z \subset E X$ be a maximal independent subset of $E X$ for the matroid $M_{2}$, that is

$$
|Z|=\rho_{2}(Z)=\rho_{2}(E X) .
$$

Let $Z^{\prime} \subset X$ be such that $Z \cup Z^{\prime}$ is a basis of $M_{2}$. Obviously, we have

$$
\rho_{2}\left(Z^{\prime}\right)=\left|Z^{\prime}\right|=r_{2}-|Z|=r_{2}-\rho_{2}(E X) .
$$

Let $W=X-Z^{\prime}$. Note that $Z \cup Z^{\prime} \subset E W$. Then

$$
C_{2} \cap C_{1}(E W, \boldsymbol{v})=\{\boldsymbol{v}\} .
$$

Namely,

$$
\boldsymbol{v} \in C_{2} \cap C_{1}(E W, \boldsymbol{v})=C_{2}(E W, \boldsymbol{v})
$$

and

Moreover, we have

$$
\operatorname{dim} C_{2}(E W, \boldsymbol{v})=r_{2}-\rho_{2}(E W)=0 .
$$

$$
\begin{aligned}
\rho_{1}(E W) & \leqslant \rho_{1}(E X)+\left|Z^{\prime}\right| \\
& \leqslant r_{1}-r_{2}+\rho_{2}(E X)-i+r_{2}-\rho_{2}(E X) \\
& \leqslant r_{1}-i
\end{aligned}
$$

that is,

$$
\operatorname{dim} C_{1}(E W, \boldsymbol{v}) \geqslant i \text {. }
$$

Take now any standard subcode of $C_{1}(E W, \boldsymbol{v})$ of dimension $i$. Then of course we have

$$
\boldsymbol{v} \in D \cap C_{2} \subset C_{1}(E W, \boldsymbol{v}) \cap C_{2}=\{\boldsymbol{v}\}
$$

and

which implies that

$$
\operatorname{Supp}(D) \subset \operatorname{Supp}\left(C_{1}(E W, \boldsymbol{v})\right) \subset \operatorname{Supp}\left(C_{1}(E X, \boldsymbol{v})\right)=X,
$$

$$
b_{i} \leqslant \operatorname{Supp}(D) \leqslant|X|=m_{i} .
$$

For the converse, let $Y \subset E$ be such that $C_{1}(Y, \boldsymbol{v}) \cap C_{2}=\{\boldsymbol{v}\}, w\left(C_{1}(Y, \boldsymbol{v})\right)=b_{i}$ and $\operatorname{dim} C_{1}(Y, \boldsymbol{v})=i$. Let $Y^{\prime}=E \operatorname{Eupp}\left(C_{1}(Y, \boldsymbol{v})\right)$. Obviously, $Y \subset Y^{\prime}$. Let $\boldsymbol{w} \in C_{1}(Y, \boldsymbol{v})$. For any $y \in Y^{\prime}, y \notin \operatorname{Supp}\left(C_{1}(Y, \boldsymbol{v})\right)$ so that $\boldsymbol{w}_{y}=\boldsymbol{v}_{y}$, and in turn $\boldsymbol{w} \in C_{1}\left(Y^{\prime}, \boldsymbol{v}\right)$. Hence, the natural inclusion $C_{1}\left(Y^{\prime}, \boldsymbol{v}\right) \subset C_{1}(Y, \boldsymbol{v})$ is actually an equality.

Let $X=E-Y^{\prime}$. Then we have

$$
|X|=\left|E Y^{\prime}\right|=\mid E\left(E \operatorname{Supp}\left(C_{1}(Y, \boldsymbol{v})\right)|=| \operatorname{Supp}\left(C_{1}(Y, \boldsymbol{v})\right) \mid=b_{i}\right.
$$

and

$$
C_{2}(E X, \boldsymbol{v})=C_{2}\left(Y^{\prime}\right)=C_{2} \cap C_{1}\left(Y^{\prime}, \boldsymbol{v}\right)=C_{2} \cap C_{1}(Y, \boldsymbol{v})=\{\boldsymbol{v}\},
$$

which implies that

$$
\operatorname{dim} C_{1}(E X, \boldsymbol{v})-\operatorname{dim} C_{2}(E X, \boldsymbol{v})=\operatorname{dim} C_{1}\left(Y^{\prime}, \boldsymbol{v}\right)-0=\operatorname{dim} C_{1}(Y, \boldsymbol{v})=i
$$

and finally

$$
m_{i} \leqslant|X|=b_{i} .
$$




\subsection{An open question concerning subcodes.}

Remark 4.2. Let

$$
b_{i}^{\prime}=\min \left\{w(D) ;\left|D \cap C_{2}\right|=1 \text {, and } D \subset C_{1} \text { is a subcode with } \operatorname{dim} D=i\right\},
$$

that is we allow the subcode to be any subcode, not only a standard subcode. Obviously, for $1 \leqslant i \leqslant R_{F}$, we have

$$
m_{i}=b_{i} \geqslant b_{i}^{\prime}
$$

It is an open question whether the last inequality is an equality. For linear codes, [18, Proposition 2] gives an analogous statement with equality. On the other hand, while $b_{i}$ and $b_{i}^{\prime}$ are both defined for $0 \leqslant i \leqslant R_{F}$, for $i>R_{F}$ it is not difficult to show that any standard subcode of $C_{1}$ will have a non-trivial intersection with $C_{2}$ but $b_{i}^{\prime}$ might be defined. Consider namely the following codes: let $A=\{0,1,2,3\}$ and $C_{1}=A^{3}$. Let $C_{2}$ and $D$ be the subcodes

$$
\{000,012,023,031,103,110,121,132,201,213,222,230,302,311,320,333\}
$$

and

$$
\{000,011,022,033,102,113,120,131,203,210,221,232,301,312,323,330\},
$$

respectively. Both subcodes have dimension 2 , while $C_{1}$ has dimension 3 . But we have $C_{2} \cap D=\{000\}$ and $\operatorname{dim} D>\operatorname{dim} C_{1}-\operatorname{dim} C_{2}$.

\section{EXTEnded Weight POlYNomials of ALmost AFFine CODES}

In [7], and in [6, p. 323], Jurrius points out that for linear block codes of length $n$ over a finite field $\mathbb{F}_{q}$, one can produce an infinite series of codes by extending the alphabet to $\mathbb{F}_{q^{s}}$, for $s=1,2, \cdots$, and nevertheless find polynomials $A_{0}, \cdots, A_{n}$, such that $A_{j}\left(q^{s}\right)$ computes the number of codewords of weight $j$, for all $s$ simultaneously, for each of $j=0, \cdots, n$. Hence knowledge of a finite number of coefficients of the $A_{j}$ compute an infinite number of weights. (A crude upper bound for this finite number is $(k+1)(n+1)$, for the length $n$ and the dimension $k$ of the code. Set $d_{0}=0$. A better bound for the finite number of coefficients of all the $A_{j}$ taken together is $1+\sum_{j=1}^{k}(j+1)\left(d_{j}-d_{j-1}\right)$.) In this subsection we will show that a corresponding result holds for almost affine codes, and we will mimick the arguments from [8, Section 3] to find weight polynomials for an infinite series of almost affine codes $C_{s}$, which we will now define.

Let $C \subset A^{n}$ for an alphabet $A$. For $s \in \mathcal{N}, C^{s}$ is a code of block length $n$ over the alphabet $A^{s}$, if an element $\left(\left(c_{1,1}, \cdots, c_{1, n}\right), \cdots,\left(c_{s, 1}, \cdots, c_{s, n}\right)\right)$ instead is interpreted as:

$$
\left(\left(c_{1,1}, \cdots, c_{s, 1}\right), \cdots,\left(c_{1, n}, \cdots, c_{s, n}\right)\right) .
$$

Let $C$ in addition be an almost affine code over $A$. It is then automatic that $\left|\left(C^{s}\right)_{X}\right|=\left(q^{s}\right)^{r}$ if $\left|C_{X}\right|=q^{r}$, for some $X \subset E$. Hence $C^{s}$ is an almost affine code over $A^{s}$, since $C$ is an almost affine code over $A$. Moreover the matroid $M_{C^{s}}=M_{C}$ since the rank functions are the same.

Let $U \subset E$, and let $\boldsymbol{c}_{\boldsymbol{Q}}$ be a fixed codeword in $C^{s}$. Similarly as in [8] we define: $S_{U}(s)=$ $C^{s}\left(U, \boldsymbol{c}_{\boldsymbol{Q}}\right)$. But, since $C^{s}$ is an almost affine code we see that $\left|S_{U}(s)\right|=\left(q^{s}\right)^{k-r(U)}$. In the next definition, there is no explicit reference to the codeword $c_{Q}$, since this is independent of the word chosen.

Definition 5.1. For each $1 \leqslant j \leqslant n$, let $A_{C, j}(s)$ be the number of codewords of weight $j$ in $C^{s}$. 
Using the exclusion/inclusion principle we get:

$$
A_{C, n}(s)=\Sigma_{i=0}^{n}(-1)^{i} \Sigma_{U} Q^{k-r(U)},
$$

where the inner sum are over all $U$ of cardinality $i$, and we obtain the same formula as in $[8$, Formula (9) p. 638]:

$$
A_{C, n}(s)=(-1)^{n} \sum_{U \subset E}(-1)^{|U|}\left(q^{s}\right)^{n^{*}(U)},
$$

where $n^{*}(Y)$ is the nullity function assosiated to the dual matroid $\left(E, \rho^{*}\right)$.

Let $a_{X, C, n}(s)$ be the number of codewords with support exactly $X$. For each $X \subset E$, we then obtain in a similar way:

Lemma 5.2.

$$
a_{X, C, n}(s)=(-1)^{n} \sum_{U \subset X}(-1)^{|U|}\left(q^{s}\right)^{n_{X}^{*}(U)}
$$

where $n_{X}^{*}$ is the nullity function of the dual rank function of the matroid $\left.\left(M_{C}\right)\right|_{X}$.

A refined study, using Proposition 2.7, also gives

Lemma 5.3. For any $U \subset X$ we have: $n_{X}^{*}(U)=n^{*}(U)$.

Combining Lemmas 5.2 and 5.3 we obtain an analogous formula as in [8, p. 638]:

Proposition 5.4. For each $j=0,1, \cdots, n$, and all $s \geq 1$ there are polynomials

$P(z)=(-1)^{j} \sum_{|X|=j} \sum_{Y \subset X}(-1)^{|Y|}(z)^{n^{*}(Y)}$ such that $A_{C, j}(s)=P\left(q^{s}\right)$.

In [8, Sections 4 and 5], one shows how this matroid expression can be expressed by $\mathbb{N}_{0^{-}}$ graded Betti numbers of the Stanley-Reisner rings of the matroid $M_{C}^{*}$ and its elongations, viewed as simplicial complexes ([8, Theorem 5.1]).

Example 5.5. Let $C$ be the almost affine code in [16, Example 2]. This is a code of rank 3 over the alphabet $\mathbb{F}_{3}^{2}$ of cardinality 9 . Its length is also 9, and its associated matroid $M_{C}$ is the non-Pappus matroid. In [5, p. 102] one calculated the polynomials $P_{j}(Q)$, for $j=0, \cdots, 9$ without relating them to any code, since $M_{C}^{*}$ is not linearly representable. The results carry over to determining the $A_{C, j}(s)$ for the non-linear almost affine code $C$, and we obtain from [5, p. 102]:

$$
\begin{gathered}
A_{C, 0}(s)=1, A_{C, 1}(s)=A_{C, 2}(s)=A_{C, 3}(s)=A_{C, 4}(s)=A_{C, 5}(s)=0, A_{C, 6}(s)=8 q^{s}-8, \\
A_{C, 7}(s)=12 q^{s}-12, A_{C, 8}(s)=3 q^{2 s}-18 q^{s}+15, A_{C, 9}(s)=q^{3 s}-9 q^{2 s}+28 q^{s}-20 .
\end{gathered}
$$

We see (by examining the exponents of the formulas for the $A_{C, j}(s)$ ) and using the next proposition, that $d_{1}=6, d_{2}=8, d_{3}=9$ for this almost affine code.

We also obtain (in the same way as shown for for linear codes in [8]):

Proposition 5.6. If $C$ is an almost affine code, we have: $d_{i}\left(C^{s}\right)=\min \left\{j \mid \operatorname{deg} P_{j} \geqslant i\right\}$, for $i=1, \cdots, k$, for all $s \in \mathbb{N}$ simultaneously.

\section{REFERENCES}

[1] T. Britz, T. Johnsen, D. Mayhew, and K. Shiromoto, Wei-type duality theorems for matroids, Designs, Codes and Crypography, vol. 62, pp. 331-341, 2012.

[2] T. Britz, T. Johnsen and J. Martin, Chains, demi-matroids and profiles, IEEE Transactions on Information Theory, vol. 60, No. 1 pp. 986-991, 2014.

[3] G.F. Forney, Dimension/Length Profiles and Trellis Complexity of Linear Block Codes, IEEE Transactions on Information Theory, vol. 40, No. 6 pp. 1741-1751, 1994. 
[4] G. Gordon, On Brylaswski's Generalized Duality, Mathematics in Computer Science vol. 6, no. 2, pp. 135-146, 2012.

[5] V. Huerga Represa, Towers of Betti Numbers of Matroids and Weight Distribution of Linear Codes and their Duals, Master's thesis in Pure Mathematics, University of Troms $\emptyset$ - The Arctic University of Norway, 2015. Available at http://hdl.handle.net/10037/7736.

[6] R.P.M.J. Jurrius, Weight enumeration of codes from finite spaces Des. Codes Cryptogr., vol. 63, pp. 321-330, 2012.

[7] R.P.M.J. Jurrius and G.R. Pellikaan, Algebraic geometric modeling in information theory, In: Codes, arrangements and matroids. Seroes on Coding Theory and Cryptology. World Scientific Publishing, Hackensack, NJ, 2001

[8] T. Johnsen, J. Roksvold and H. Verdure, A generalization of weight polynomials to matroids, Discrete Mathematics, vol. 339, No, 2, pp. 632-645, 2016.

[9] T. Johnsen, H. Verdure, Hamming weights of linear codes and Betti numbers of Stanley-Reisner rings associated with matroids, AAECC, vol. 24, pp. 73-93, 2013.

[10] Johnsen, T., Verdure, H., Generalized Hamming weights for almost affine codes, IEEE Trans. Inform. Theory, 63, no. 4, 1941-1953 (2017)

[11] Johnsen, T., Verdure, H.: Flags of almost affine codes and the two-party wire-tap channel of type II, Designs, Codes and Crypography,https://doi.org/10.1007/s10623-017-0438-1, 2017.

[12] Johnsen, T., Verdure, H.: Relative Generalized Hamming Weights and Extended Weight Polynomials of Almost Affine Codes, in Coding Theory and Apllications, Proceedings of the 5'th International Castle Meeting, Vihula, Estonia, August 2017, LNCS 10495, 207-16 (2017).

[13] Z. Liu, W. Chen, Yuan Luo, The relative generalized Hamming weight of linear q-ary codes and their subcodes,Designs, Codes and Cryptography, Volume 62, pp 111-23 (2008).

[14] Y. Luo, C. Mitrpant, A.J. van Vinck, and K. Chen, Some New Characters on the Wire-Tap Channel of Type II, IEEE Transactions on Information Theory, vol. 51, No. 3, pp. 1222-1229, 2005.

[15] J.G. Oxley, Matroid theory, Oxford university press, 1992.

[16] J. Simonis and A. Ashikhmin, Almost Affine Codes, Des. Codes Crypogr., vol. 14, pp. 179-197, 1998.

[17] V.K. Wei, Generalized Hamming weights for linear codes, IEEE Trans. Inf. Th., vol. 37, No. 5, pp. 1412-1418, 1991.

[18] Zhujoan Zhuang, Bin Dai, Yuan Luo, A.J. Han Vinck, On the relative profiles of a linear code and a subcode, Designs, Codes and Cryptography, August 2014, Volume 72, Issue 2, pp. 219-247

Department of Mathematics and Statistics, Uit-The Arctic University of Norway N-9037 Troms $\varnothing$, Norway

E-mail address: trygve.johnsen@uit.no

Department of Mathematics and Statistics, Uit-The Arctic University of Norway N-9037 Troms $\emptyset$, NorwaY

E-mail address: Hugues.Verdure@uit.no 